\title{
Cuidados intensivos pediátricos: pasado, presente $y$ futuro
}

\author{
JORGE ROQUE E. ${ }^{1}$ \\ 1. Médico jefe UCI Pediatria, Clínica Alemana.
}

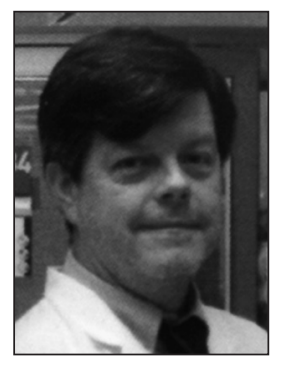

Para muchos la primera reanimación bocaboca descrita en la historia de la humanidad aparece en el libro segundo de los Reyes de la Biblia, donde se relata la reanimación de un niño Sunamita por parte del profeta Eliseo, fechada aproximadamente alrededor del año 700 A.C. "El profeta se subió a la cama y se acostó sobre el niño colocando su boca, ojos y sus manos contra las del niño y estrechando su cuerpo contra el suyo y lo hizo más de una vez, y el niño fue adquiriendo color y estornudó 7 veces."

Hubo de pasar muchísimo tiempo en la historia de la humanidad, antes de que los primeros ejemplos de unidades de cuidados intensivos (UCI) en el mundo aparecieran. Recién en 1854 durante la guerra de Crimea en la que Inglaterra, Francia y Turquía declararon la guerra a Rusia, aparece la experiencia de Florence Nightingale y más de 38 voluntarios capacitados por ella, que se dedicaron a atender a los soldados heridos en una unidad de pacientes graves diseñada por esta enfermera y escritora, logrando bajar la mortalidad desde un $40 \%$ a tan sólo un $2 \%$.

Posteriormente, en EE.UU., Walter Edward Dandy, quien fuera un pionero de las operaciones neuroquirúrgicas, observó la necesidad de controlar el despertar de la anestesia en los pacientes quirúrgicos, lo que lo motivó a impulsar la creación de salas adyacentes a los pabellones quirúrgicos, denominadas "recovery rooms". Fundó así la primera UCI de recuperación de anestesia de la que se tenga referencia, en el hospital John Hopkins en Baltimore en 1920.

El desarrollo consecuente de estas unidades estuvo asociado al avance paralelo de la cirugía y la anestesiología, que experimentaban grandes progresos en los campos de la cardiocirugía e intervenciones de tórax. No fue sino hasta la década de los 50 en que se produjeron las epidemias de poliomielitis que se vio un desarrollo aparte de estas unidades para la atención de pacientes con insuficiencia respiratoria, y que tratados en ventiladores a presión negativa intermitente dentro de un recipiente metálico (pulmón de acero) presentaban una mortalidad cercana al 90\%. Fue así como en 1952, el Dr. Bjørn Ibsen, un anestesista danés, decidió tratar estos pacientes con traqueotomía infralaríngea y Ventilación a Presión Positiva manual con bolsa de resucitación. Con este nuevo método, se logró un descenso de la mortalidad al 45\%. Al mismo tiempo, Ibsen pudo objetivar los beneficios de tener en forma permanente un área específica dotada de personal entrenado y multidisciplinario para el manejo adecuado de los pacientes críticos. De esta forma logró establecer la primera UCI general en el Hospital Kommune (Copenhagen) en 1954. Cabe destacar que el "personal entrenado" al que recurrió inicialmente el Dr. Ibsen, fueron estudiantes de medicina y monjas, quienes ad- 
mirablemente asumieron el riesgo de adquirir la poliomielitis para ventilar manualmente a sus pacientes durante semanas y así salvarlos de una muerte casi segura.

Un nuevo hito lo marcaria el Dr. Peter Safar, médico nacido en Austria, quien emigró a los Estados Unidos después de permanecer en un campo de concentración nazi. Se graduó como médico anestesista y es conocido como el "padre de la reanimación moderna". Propuso la secuencia del ABC primario en la reanimación, creando la técnica del boca a boca, respiración artificial y masaje cardíaco externo. Apoyó decididamente al desarrollo del concepto de soporte vital en los pacientes críticamente enfermos e inició los estudios de la hipotermia terapéutica dentro de la reanimación avanzada. Fue él quien acuño el término "intensive care medicine" y estableció la primera Unidad de Cuidados Intensivos de EE.UU en 1962, en Baltimore. También fundó la Asociación Mundial de Medicina de Urgencias y fue co-fundador de la SCCM (Society of Critical Care Medicine), en 1970 en conjunto también con otros 2 grandes del intensivismo mundial, Max Harry Weil y William Shoemaker.

Como ha ocurrido la mayor parte del tiempo a lo largo de la historia de la medicina moderna, el desarrollo de la especialidad en pediatría fue algo más rezagado con respecto a los adultos, siendo en la década de 1960 en que se comienza la atención del niño críticamente enfermo. Correspondió a la neonatología el privilegio de ser la pionera en la atención intensiva en pediatría, centrándose primeramente en el distres respiratorio neonatal. Fue en Liverpool (Inglaterra), Gothemborg (Alemania), Philadelphia y Pittsburg (EE.UU.) donde se crearon las primeras unidades de Cuidados Intensivos Pediátricos.

En Chile el desarrollo de estas unidades también fue algo más tardío, ya que las grandes patologías predominantes en la pediatría en aquellos años eran la diarrea y las infecciones respiratorias bajas graves. Ambas patologías concentraban los limitados recursos de las autoridades tratándolas con exitosos programas de atención social (campañas contra la desnutrición infantil, controles sanitarios, disponibi- lidad de agua potable, vacunas, etc.), y terapias muy efectivas y de bajo costo relacionadas con la hidratación en el caso de las diarreas y con el uso de antibióticos en el caso de las neumonías. Luego eran menos los que finalmente requerían de atención más compleja, y al mismo tiempo se lograban descensos significativos de la mortalidad infantil.

Al igual que en el extranjero, en 1958, aparecía la primera UCI pediátrica (UCIP) cardiológica en el hospital Luis Calvo Mackenna, como una sala de recuperación de las cardiocirugías que se estaban desarrollando, y a finales de la década de los años 60 y principios de los 70 , se gestó la primera unidad de cuidados intensivos de pediatría polivalente, liderada por el Dr. Patricio Olivos en compañía del Dr. Jaime Cordero, con una mirada de futuro de lo que se venía en el desarrollo de las diferentes subespecialidades de la pediatría, muchas de las cuales requerirían del apoyo de las UCIs pediátricas. En 1966 el Hospital Roberto del Río creó una sala de emergencias respiratorias, adquiriendo su primer ventilador mecánico marca Bird Mark 8 ("pájaro verde"). Esta unidad monovalente respiratoria poco a poco fue recibiendo pacientes con otras patologías, y durante su desarrollo contó con el gran aporte del Dr. Antonio Garate. En 1998 la sala paso a cargo de los primeros intensivistas y pasó a llamarse Unidad de Cuidados especiales (polivalente) a cargo del Dr. Carlos Casar. Casi paralelamente se desarrollaban la UCIPs de los hospitales Sótero del Río y Exequiel González Cortés. En región, la primera UCI pediátrica aparece en el Hospital Deformes de Valparaíso, a cargo del Dr. Gustavo Ríos en 1981 y fue en dicho centro donde se realizó la primera Hemofiltración en el país en el año 1987.

Sin embargo, la aparición sucesiva de las UCIPs en Chile no fue producto de una política ministerial ajustada a la realidad sanitaria y planificada, si no que más bien fue produciéndose un desarrollo desordenado de las unidades, de acuerdo a loables iniciativas de los pediatras y a los recursos disponibles en cada hospital. Un grupo entusiasta de pediatras organizó el comité de intensivistas pediátricos en 1988, al alero de la sociedad Chilena de Pediatría. Entre ellos estaban los Drs. Patricio Romero, Jaime 
Cordero, Carlos Casar y Mario Cerda. En la década de los 90 se hacía evidente la existencia de UCIPs en casi todos los hospitales con servicios pediátricos especialmente en Santiago, algunas ciudades de provincia, hospitales universitarios y centros privados importantes, pero aún la mayoría eran pequeñas, y cada una con su personal y residencia completa, sin concentrar recursos, con "casuísticas repartidas" y con médicos autodidactas en su mayoría, pero muy entusiastas y entregados a su trabajo de intensivistas. Los grupos líderes en Santiago tomaban contacto con los mejores centros en Europa y EE.UU., se producían las primeras estadías de perfeccionamiento en aquellos centros que lideraban el intensivismo mundial y se estrechaban contactos. Se impactaba positivamente en la mortalidad, y se hacía evidente que en invierno la demanda por camas críticas prácticamente colapsaba estas unidades. Los intensivistas pediátricos se organizaban, cada centro en forma aislada organizaba cursos con invitados internacionales y se encontraban periódicamente en reuniones científicas, donde se discutía y compartía experiencia en temas propios de la especialidad pero también las contingencias a las que estaban sometidas las UCIPs.

Por entonces, apareció el primer programa de formación de medicina intensiva pediátrica de Chile en la Pontificia Universidad Católica de Chile el año 1996, siendo su primer alumno el Dr. Andrés Castillo, y 2 años después se sumó el programa de la Universidad de Chile.

El año 1997 se realizó el III Congreso Latinoamericano de Emergencias y Cuidados Intensivos Pediátricos, en Viña del Mar organizado por el Dr. Gustavo Ríos.

El año 2000 marca 3 hitos, primero se transformó el comité en una rama de cuidados intensivos de pediatría de la Sociedad Chilena de Pediatría, encabezada por una directiva elegida por voto entre los intensivistas pediátricos, cuyo primer presidente no he podido dilucidar, pero sí sé que contó entre otros con los primeros fundadores del comité más los Drs. Ricardo Ronco, Cristian Valverde, Bettina Von Dessauer, Roberto Dalmazzo y Gustavo Ríos y se comenzó a emitir un boletín de la rama; segundo, la Comisión Nacional de Acreditación de Especialidades Médicas (CONACEM) reconoció la subespecialidad de intensivo de pediatría; y tercero, se realizó la primera Jornada Nacional de Cuidados Intensivos Pediátricos de la recién constituida rama de Cuidados Intensivos pediátricos organizada por las UCIPs del hospital Roberto del Río (Dra. B. Von Dessauer) y San Juan de Dios (Dr. J. Roque), las cuales se repitieron por 2 años seguidos, hasta desembocar en el primer Congreso de Intensivo Pediátrico de Chile en Puerto Montt el año 2003. Nuevamente el año 2008 se realizó el IX Congreso Latinoamericano de Cuidados Intensivos Pediátricos en Viña del Mar, organizado por la Rama de Cuidados Intensivos presidida ese año por el Dr. R. Ronco.

Han pasado varios años de desarrollo sostenido de estas jóvenes unidades, no exentas de problemas y complejidades propias de una subespecialidad reciente y sometida a rápidos y grandes cambios tecnológicos. Hasta ahora, la pequeña pero sólida comunidad de intensivistas pediátricos ha logrado sortear con éxito los desafíos que se han ido planteando a lo largo de su historia, y de ser unidades pequeñas, separadas, autodidactas y de escasos recursos, han pasado a ser unidades mucho más complejas, que manejan presupuestos importantes, conectadas en red asistencial, sometidas a una alta demanda por las expectativas de la población, con médicos intensivistas con formación en la subespecialidad en Chile y en el extranjero, aún mas sobrecargadas de trabajo, con una tecnología costosa, compleja de incorporar y manejar, sometidas a difíciles dilemas éticos por sus pacientes y sus familias, escrutadas por los medios atentos a las noticias fáciles y sensacionalistas, y con un público más empoderado de sus derechos.

Vivimos tiempos en que se ha logrado bajar sustancialmente la mortalidad infantil, los pacientes que sobreviven, en muchos casos pasan a formar parte de la creciente población pediátrica con enfermedades crónicas, que periódicamente se hospitalizan en las UCIPs o que definitivamente se quedan por necesidad de tecnología de soporte vital por largos períodos de tiempo (meses, años), restándole capacidad de respuesta a las unidades que ven 
disminuidos sus cupos de camas para pacientes graves.

Un importante protagonismo han adquirido los centros de salud privados, que han aportado incorporando costosas técnicas y procedimientos en intensivo que nos han permitido ir casi a la par con el conocimiento en el mundo y que posteriormente se han equiparado en el sistema público. Ventilación de alta frecuencia, nuevos ventiladores mecánicos de última generación, monitorización cada vez más exacta y menos invasiva, terapias de reemplazo renal agudo, dispositivos y procedimientos intravasculares para niños cada vez más pequeños, manejo de trasplantes complejos, monitorización de drogas en sangre, ECMO neonatal y pediátrico, nuevas drogas y productos de ingeniería genética, exámenes de laboratorio cada vez más exactos, etc. Pero todo eso también ha traído aparejado el aumento de los costos, y nos plantea en el día a día hasta donde podemos llegar, cuanto es justo, cuanto es racional, cuanto es correcto, cuanto es ético cuando se trata de la vida de un niño y de muchos otros que también requieren de los siempre limitados recursos.

Este año se realizará el décimo congreso nacional de cuidados intensivos de pediatría en Santiago, y será una oportunidad para que rescatemos nuestra historia, que está plagada de entrega, ingenio y sacrificios continuos de los intensivistas pediátricos. Una historia que nos permita proyectarnos hacia el futuro, que genere la reflexión acerca de los nuevos desafíos que se nos presentan:

1. Especialidad en falencia. Según datos de la rama de cuidados intensivos de pediatría, sólo el 29\% de los residentes de las UCIPs públicas en Chile son intensivistas acreditados, $15 \%$ de los cargos no están cubiertos, 36\% de las UCIPs comparten sus residentes con otros servicios. Sólo 3 universidades tiene un programa de formación de subespecialistas en intensivo pediátrico, y desafortunadamente los estímulos económicos y el estilo de vida de los intensivistas ha hecho disminuir dramáticamente las postulaciones de profesionales jóvenes, que ven en esta especialidad un camino de menores remuneraciones y más sacrificio que la de otras subespecialidades de la pe- diatría. Aquí se requiere una mirada de país que estimule nuevas vocaciones si desean que esta década no retrocedamos todo lo ganado en los años previos.

2. Uso racional de los recursos, lo que implica contar con profesionales idóneos, que busquen la excelencia en su trabajo a través del perfeccionamiento personal continuo y la calidad en la atención de sus pacientes. Que no se "encandilen" con nuevas terapias y que sepan derivar oportunamente a centros más especializados. No todas las UCIPs tienen que tener la misma complejidad, de hecho algunas se especializan en resolver problemas más agudos, mientras que otras concentran pacientes más complejos y "costosos" que requieren más tiempo y de equipos multidisciplinarios para resolver las patologías o estabilizarlas (niños con necesidades especiales de atención en salud (NANEAS) descompensados). Aquí se requiere una mirada de país, para planificar y distribuir adecuadamente los recursos (que siempre son escasos) y las tareas de cada UCIP.

3. Realizar más trabajos colaborativos entre las diferentes UCIPs, lo que requiere una rama fuerte e intensivistas generosos $\mathrm{y}$ comprometidos en el trabajo de investigación clínica. Los medios tecnológicos de comunicación permiten hoy intercambio de datos con más fluidez y análisis estadísticos más certeros. Esta es una tarea de los intensivistas.

4. Desarrollo de áreas en falencia, ayudando a generar centros de referencia regionales, traspasándoles experiencias, tecnologías nuevas, estimulando el intercambio de profesionales, mejorando las comunicaciones entre los centros, desarrollando normas y guías de atención conjuntos, realizando cursos con temas específicos, en fin, compartiendo.

5. Colaboración público privada, que en un país pequeño como el nuestro que no se puede dar el lujo de desperdiciar recursos, deben existir puentes de comunicación y desarrollos complementarios, que al final no dejen a ningún niño sin la atención que requiera. 
6. Definición de una política con mirada de país para tratar en un espacio adecuado y con dignidad a los pacientes con NANEAS que requieren de tecnología o soporte vital permanente para que no estén ocupando camas en las UCIPs donde están expuestos a complicaciones infecciosas.

Al final, vuelve a mi memoria el relato de
Ibsen, y sus voluntarios, monjas y estudiantes, que por semanas expusieron sus vidas para salvar otras, inspirándonos a ejercer nuestra profesión con sentido de trascendencia. Porque las cosas valen la pena no sólo por lo tangible, sino que también por otros valores que me hacen mejor persona a mí, a mi familia, a la sociedad, y a aquellos con quienes compartimos nuestro trabajo. 